\title{
Free Energy Self-Averaging in Protein-Sized Random Heteropolymers
}

\author{
Jeffrey Chuang ${ }^{1}$, Alexander Yu. Grosberg ${ }^{2,3}$, and Mehran Kardar ${ }^{1,4}$ \\ ${ }^{1}$ Department of Physics, Massachusetts Institute of Technology, Cambridge, Massachusetts 02139 \\ ${ }^{2}$ Department of Physics, University of Minnesota, Minneapolis, Minnesota 55455 \\ ${ }^{3}$ Institute for Biochemical Physics, Russian Academy of Sciences, Moscow 17r234, Russia \\ ${ }^{4}$ Theoretical Physics, University of California, Santa Barbara, California 93106
}

\begin{abstract}
Current theories of heteropolymers are inherently macroscopic, but are applied to folding proteins which are only mesoscopic. In these theories, one computes the averaged free energy over sequences, always assuming that it is self-averaging - a property well-established only if a system with quenched disorder is macroscopic. By enumerating the states and energies of compact 18,27, and 36mers on a simplified lattice model with an ensemble of random sequences, we test the validity of the self-averaging approximation. We find that fluctuations in the free energy between sequences are weak, and that self-averaging is a valid approximation at the length scale of real proteins. These results validate certain sequence design methods which can exponentially speed up computational design and greatly simplify experimental realizations.
\end{abstract}

Protein folding remains one of the most challenging

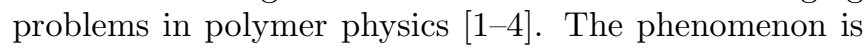
straightforward - at low temperature a heteropolymer chain freezes into a single configuration. However, the relationship between a chain's monomer sequence and the thermodynamics of its transition is complex. As a result, current theories of heteropolymer freezing resort to certain assumptions which have not been adequately tested, one of the most basic being self-averaging of the free energy.

Self-averaging is a property of many disordered systems, stating that the free energy of a system of size $N$ with quenched disorder is independent of the particular realization of the disorder, to within variations of order of $\sqrt{N}$, which are relatively negligible as $N \rightarrow \infty$. This property can be rigorously proved for a broad range of models in macroscopic disordered systems [5]. Fundamentally, it stems from the independence of sub-regions in the $N \rightarrow \infty$ thermodynamic limit. In the context of heteropolymers, it states that a random heteropolymer's free energy is independent of its sequence, i.e.

$$
F(\text { seq }, T) \simeq\langle F(\text { seq }, T)\rangle_{\text {seq }}
$$

where $\langle\cdots\rangle_{\text {seq }}$ indicates an average over sequences. There have been some proofs of self-averaging for certain heteropolymer models in the $N \rightarrow \infty$ limit [6]. Proteins, however, are mesoscopic objects, and it is unclear whether self-averaging applies at the lengths of $N$ not more than several hundred monomers found in proteins.

Self-averaging in heteropolymers is important for two main reasons. First, it is relevant to the theoretical understanding of protein folding. Starting from [2,3], key modern theories of heteropolymers, reviewed recently in [1], compute the averaged free energy of the system, implicitly neglecting sequence-dependent variations in the manner of Eq. (1). In particular, self-averaging is an element of the replica method, and is used in the derivation of the Random Energy Model for heteropolymers
22. Second, self-averaging is an important assumption of certain sequence design methods such as "imprinting" [7] and "sequence selection [8]," ideas from which have been used for de novo protein and ligand design [9]. These methods have proven useful both experimentally [10] and computationally. Unfortunately, computational design methods that do not assume self-averaging 11] require vastly more calculation time. To design a heteropolymer sequence to fold into a certain conformation $*$ at temperature $T$, one should minimize the quantity $E(s e q, *)-F(s e q, T)$ over all sequences. If selfaveraging is not assumed, one must calculate the energy of all conformations for each sequence tested to determine $F(s e q, T)$. However, if self-averaging is valid, then the $F$ term can be ignored and design can be carried out by evaluating the energy of each sequence in just the one conformation $*$. This exponentially speeds up the design procedure.

In vitro experiments have not yet provided sufficient evidence to verify self-averaging in random peptide chains. In the experiments that have studied random amino acid sequences, there have not been any obvious trends in the behavior [12], due to the difficulty of such experiments and consequent lack of data. However, using a computer simulation, we are able to sample many more sequences than can be analyzed feasibly in vitro, and thus determine whether the property of free energy self-averaging over sequences is valid for heteropolymers. We perform a scaling comparison of the exact free energy and other parameters for several three-dimensional lattice heteropolymers of different size. We then extrapolate our data to determine the validity of free energy self-averaging for protein-sized polymers.

In order to study the thermodynamics of random heteropolymers, we perform an exact lattice enumeration of the states of compact polymer chains of several different lengths. For each length, we examine many random sequences made up of two monomer species, $\alpha$ and $\beta$. All included sequences have the same number of monomers 
of type $\alpha$ and type $\beta$, so as to remove any concentration dependence. For each sequence, we evaluate its energy in all possible compact conformations. Using this information, we then calculate the free energy $F(T)$ of each sequence. To determine whether self-averaging is valid, we first compare the average and the standard deviation of the free energy over the examined sequences, and then examine the dependence of these quantities on the chain length $N$. Our method of enumeration is in contrast with other works which use Monte Carlo sampling of states to determine averaged thermodynamic properties [1, 13]. By doing a full enumeration, we are able to separate thermodynamic properties of the system from kinetic effects. In practice, the method is similar to the procedure used in studies of designability 14, although we only examine a finite sample of sequences, rather than testing every possible one. In our case, the focus is the complete free energy versus temperature curve, rather than just the ground state conformation for each sequence.

We use a standard model in which monomers are placed at lattice positions $r_{i}$, and subject to an energy

$$
E=\sum_{i<j}^{N} B_{s_{i} s_{j}} \Delta\left(r_{i}-r_{j}\right)
$$

where $i$ and $j$ run over the monomers in the chain, and $s_{i}$ indicates one of the species ( $\alpha$ or $\beta$ ) of the monomer $i$ for a particular sequence $\left\{s_{i}\right\}$. Contact interactions are enforced by setting $\Delta\left(r_{i}-r_{j}\right)=1$, if the $r_{i}$ and $r_{j}$ are on neighboring lattice points, and 0 otherwise. Interactions between neighbors along the chain are not included as their total only provides a reference point for the other energies. The interactions between monomer species are tabulated in a matrix $\widehat{B}$ having mean interaction $B=0$, and standard deviation $\delta B=1$. These values are weighted according to the fraction of monomers of each species in the system, i.e. $B=\sum_{k, l} p_{k} B_{k l} p_{l}$, and $\delta B^{2}=\sum_{k, l} p_{k}\left(B_{k l}-B\right)^{2} p_{l}$, with $k$ and $l$ taking on the monomer species types $\alpha$ and $\beta$. With these constraints, homopolymer effects are removed and the freezing temperature of the system should be of the order of $\delta B=1$. We first focus on Ising-type interactions, in which $B_{\alpha \alpha}=B_{\beta \beta}=1$ and $B_{\alpha \beta}=B_{\beta \alpha}=-1$.

The restriction to compact conformations is partly dictated by computational constraints, and allows us to fully enumerate much larger values of $N$ than would be possible otherwise. This choice is also physically justified since, according to the molten globule model of freezing [1], the available states of proteins at the freezing transition are mostly compact. Furthermore, all such compact configurations have the same number of contacts, and therefore energy differences between configurations are only due to heteropolymeric contributions. We have selected a compact state with interactions switched off $(B=0)$ as our reference zero energy state (i.e. a noninteracting compact homopolymer). With this choice, the fluctuation in free energy over sequences is the heteropolymeric quantity important to sequence design.
We enumerated chains of length $18(3 \times 3 \times 2), 27$ $(3 \times 3 \times 3)$, and $36(3 \times 3 \times 4)$. The ratios $\alpha: \beta$ in these chains were $9: 9,14: 13$, and $18: 18$, respectively. We restricted our study to a set of 500 sequences each for 18 mers and 27 mers, and 120 sequences for 36 mers for reasons of computational tractability. The enumeration algorithm followed the procedure of Pande et al [17]. Computations were carried out on two pentium-II computers and on a cluster at the University of Minnesota Supercomputing Institute over a period of a few months.

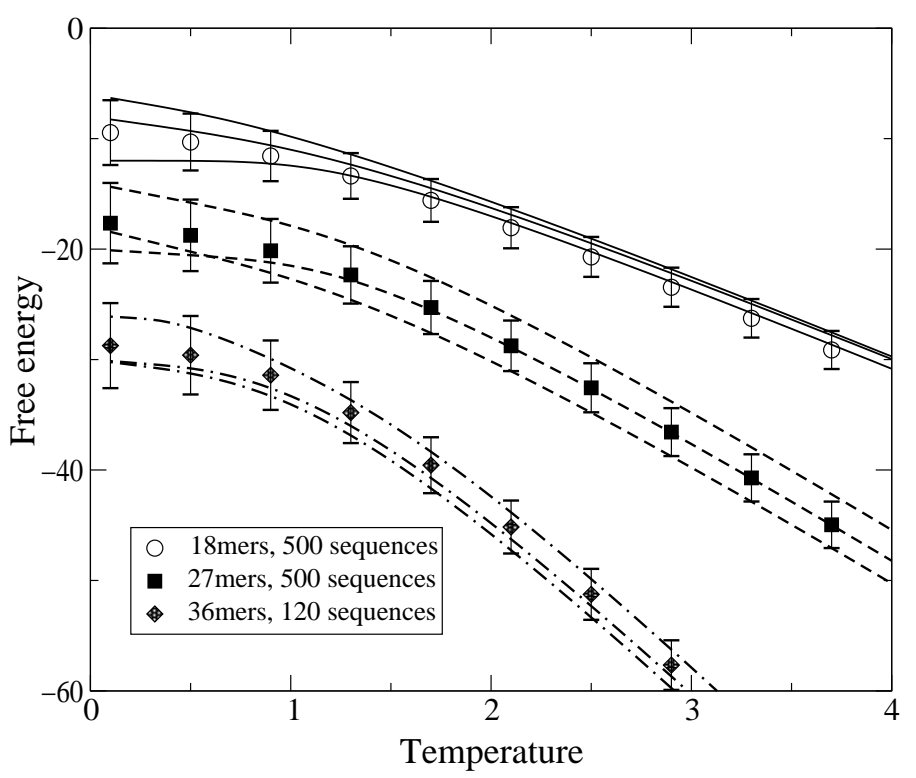

FIG. 1. Sequence dependent free energies versus temperature for three different lengths $N$. The symbols and errorbars indicate the averaged free energy, and its standard deviation. The solid lines are the free energy curves for a few sample sequences. Variations in the free energy are small compared with its absolute value at all temperatures.

Given the limitations of a lattice simulation, we cannot address questions that depend on the microscopic details of real proteins, and instead focused on general trends which should be robust across different polymer models. The basic test of self-averaging is whether sequencedependent variations in thermodynamic quantities are significant. Let us first review the general features of the free energy and its sequence dependent fluctuations: For any sequence, the free energy $F=E-T S$, is expected to be linear in temperature at both high and low temperatures. At high temperatures, all states are accessible, and the free energy is dominated by $T S_{\text {all }}(N)$, where $S_{\text {all }}(N)$ is the logarithm of the number of compact conformations of length $N$. Below its freezing temperature, the free energy is controlled by the lowest energy states, with a much smaller (possibly zero) slope of temperature dependence given by the degeneracy of these states. 
At these low temperatures, the entropy component of the free energy is expected to depend strongly on the sequence [1], though this contribution is small compared to the energy component, which should be proportional to $N$, and equal to within $\sqrt{N}$ for all sequences [1]. Fig. (1) shows a few sample sequences that illustrate this behavior. The error bars indicate the standard deviation of the free energy $\delta F$ at each temperature, calculated over the ensemble of sequences. One can see that the behavior is as expected: at high temperature the curves are parallel and linear in $T$; at low temperature the sequence dependence is more important - in particular below the freezing temperature, where the slopes of the curves change around $T \approx 1$.

More importantly, Fig. (1) shows that the variations in the free energy across sequences are significantly less than the absolute value of the free energy. In other words, the sequence dependent fluctuations are weak and Eq. (1) is a good approximation. At higher temperatures, the relative fluctuations become even less significant, because of the greater importance of the $T S$ contribution.

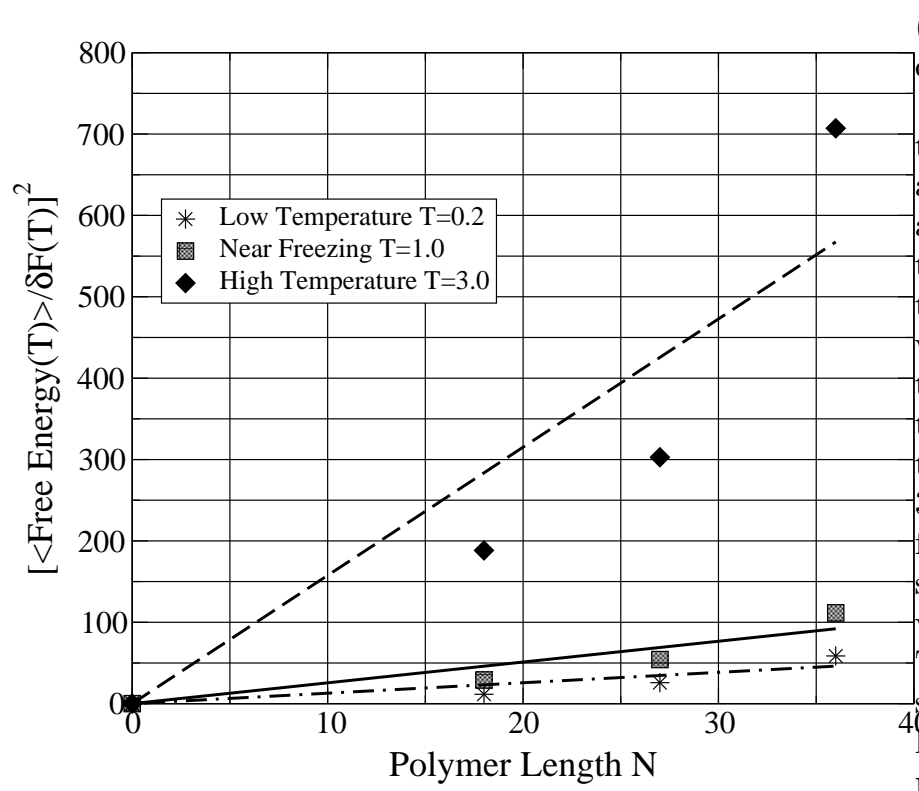

FIG. 2. The quench-averaged square of the free energy divided by the free energy variance, as a function of polymer length, at 3 different temperatures. The large values of this quantity, as well as its increasing trend with $N$, imply that the free energy variations between sequences will be insignificant for polymers of hundreds of monomers, the length scale of proteins.

In Fig. (2) we test self-averaging trends by considering the size dependence of the relative variations in the free energy. At this stage we should clarify what we mean by self-averaging, i.e. the conditions that justify the heteropolymer theories, as well as the fast methods of se- quence design. Heteropolymer theories rely on Eq. (1), i.e. that fluctuations are small. More precisely, the standard deviation among sequences $\delta F(T)$ must be much less than the average value $\langle F(T)\rangle$. Design algorithms, on the other hand, are used to find sequences for which $E$ and $F$ are of the same magnitude. Therefore, the fast design method of minimizing just $E$ is a sufficient procedure so long as sequence-dependent fluctuations of $F$ are much smaller than $F$ itself, and hence much smaller than the sequence dependencies selected into $E$. Thus the fast design methods will be justified under the same condition of $\langle F\rangle / \delta F \gg 1$. Another trend that we can look for is whether $\langle F\rangle / \delta F$ is increasing with $N$. If this is true, then proteins, which have values of $N$ about an order of magnitude larger than what we test, should have even better self-averaging than our lattice models. We indeed expect such a trend as larger values of $N$ should include more independent subregions, although this notion is imprecise and there should be finite size effects 13.

As Fig. (2) shows, the results strongly support selfaveraging: $\langle F\rangle / \delta F \gg 1$ for all the data points at all temperatures and polymer lengths. Furthermore, $\langle F\rangle / \delta F$ is increasing in $N$, which shows that the self-averaging is even better justified for larger protein-sized polymers. (The reason for plotting in the square of $\langle F\rangle / \delta F$ has to do with extensivity, as discussed below.)

Figure (2) is the main result of this paper. It shows that even for chains as short as 18 monomers, selfaveraging holds for binary sequences. As real proteins are of the order of 100 amino acid units, it seems likely that self-averaging will be valid for them as well. Although we are only showing data for Ising interactions, we believe these results to be valid for all $2 \times 2$ interaction matrices with zero mean and unit variance. We have tested several other matrices, using the simple parameterization suggested in [15, 16], with $\theta=0, \pi / 8, \pi / 4$, and $3 \pi / 8$. All of these matrices show similar trends, and in fact have even higher values for $\langle F\rangle / \delta F$ than those presented here. This strongly suggests that self-averaging is valid independent of the choice of the interaction matrix. These results should generalize to chains with more possible monomers (e.g. proteins), so long as the chains are 40 long enough that the mean and variance of the monomermonomer interactions accurately describe the chain energies. That is, the number of contacts in a conformation should be at least of the order of the number of possible monomer-monomer interactions. This would be true, if, as is commonly accepted, only a few different interactions (e.g. hydrophobicity) are significant - though the actual number has been the subject of some scrutiny [15].

A secondary issue related to self-averaging is extensivity. Self-averaging is traditionally derived from the idea that sub-regions of the system behave independently [5]. The free energy of the complete system is approximately equal to the sum of the free energies for many small subregions. Each subregion contains a random realization of local disorder, and if the system is large, the sum of the free energy over subregions will be independent of the 
overall quenched disorder. A consequence of such independence of subregions is that both the free energy, and its variance, will be extensive, i.e. linear in $N$ as $N \rightarrow \infty$.

Figure (3) tests the extensivity of the free energy at three different temperatures; below, close to, and above, the freezing transition. As a rough guide, we have included linear fits that pass through the four points at $N=0,18,27$, and 36 . Note that while the free energy is zero at $N=0$ (a polymer of length zero has no energy or entropy), the asymptotic linear limit for large $N$ does not have to pass through this $N=0$ point because of subleading surface terms. In order that the different temperatures may be better compared, the free energies have been divided by temperature, and compared with their infinite temperature value of $S_{\text {all }}(N)$. The results at $T=3$ are practically indistinguishable from $S_{\text {all }}(N)$, and in fact the dependence on $N$ shows similar trends at all three temperatures. It was shown by Pande et al. [17] that that $S_{\text {all }}(N)$ has a good linear form when $N$ is extended to lengths as short as $N=48$. Because of this, we expect extensivity to improve when $N$ is marginally larger than what we have tested here.

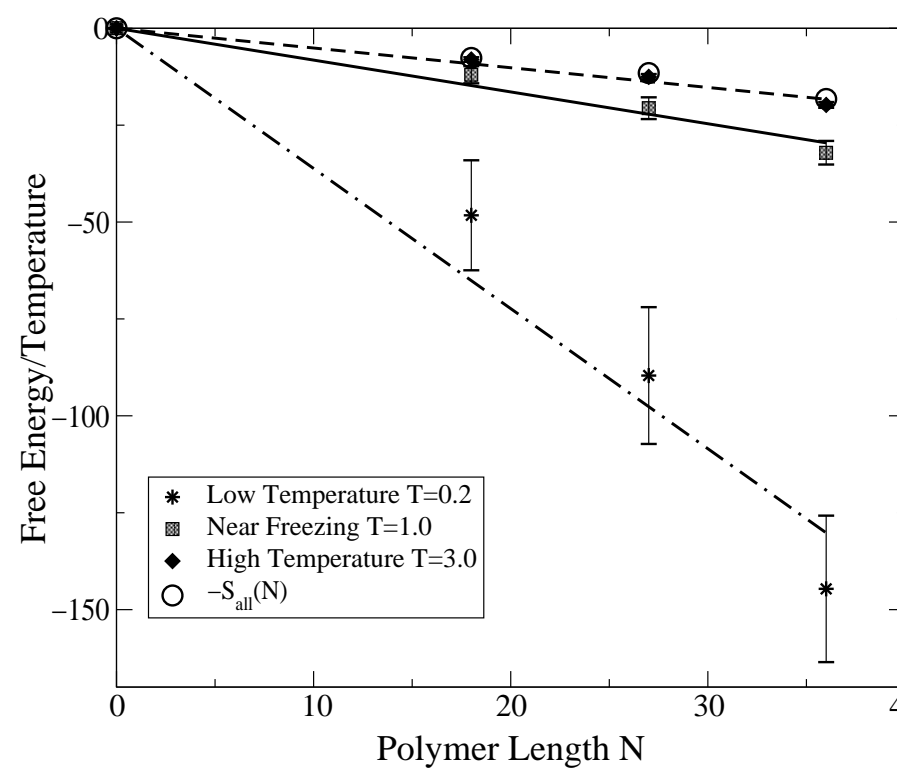

FIG. 3. The dependence of the free energy on $N$, below, near, and above the freezing temperature. Each curve has been divided by the corresponding temperature, so as to compare with the infinite temperature limit provided by the (logarithm of) the number of configurations. The deviations from linearity indicate the importance of finite size effects for protein sized heteropolymers.

Since for a globule made up of independently contributing subunits we would expect the variance in the free energy to scale as $N$ as well, the quantity $\langle F(T)\rangle^{2} / \delta F(T)^{2}$ should be proportional to $N$. Figure (2) suggests that this may be the case at least at low temperatures. The available data, however, have an upward curvature, and the values at $N=36$ are systematically higher than our attempted linear fits. It may well be that, as in the case of entropy calculation in Ref. [17], the results for $\langle F(T)\rangle^{2} / \delta F(T)^{2}$ become linear at marginally higher values of $N$. Despite these deviations from the expected asymptotic extensivity, the large magnitude of the plotted values justify self-averaging according to Eq. (1). It is indeed the very deviations from the asymptotic behavior at these smaller sizes that necessitated the current study, as it indicates that protein sized objects are not quite extensive in the thermodynamic sense.

The main conclusion of this work is that sequencedependent fluctuations in the free energy of random heteropolymers are small, even at values of $N$ as low as $N=18$. Qualitatively speaking, this means that all random sequences have nearly the same free energy. There are also indications that the fluctuations decrease in importance as $N$ increases. These facts together imply that self-averaging will be a good approximation for proteinsized heteropolymers. Although there are deviations from thermodynamic extensivity at this length scale, the key property of self-averaging is verified. This latter property is important to sequence design algorithms. Our results show that sequence design can be carried out without having to calculate the energy of each tested sequence in all conformations. Instead, one need only calculate the energy of each sequence in the desired conformation. This shortcut vastly reduces the necessary -computation time.

We would like to acknowledge illuminating discussions with the late Professor Toyoichi Tanaka about this work, as well as inspiration and encouragement from him during the years of collaboration. JC acknowledges support from an NSF graduate fellowship. MK is supported by NSF grants DMR-98-05833 (at MIT) and PHY99-07949 (at ITP).

[1] V. S. Pande, A. Yu. Grosberg, and T. Tanaka, Reviews of Modern Physics 72, 259 (2000).

[2] E. I. Shakhnovich and A. M. Gutin, Biophysical Chemistry 34, 187 (1989).

[3] J. D. Bryngelson and P. G. Wolynes, Proc. Natl. Acad. Sci. USA 84, 7524 (1987).

[4] K. A. Dill, Protein Science 8, 1166 (1999).

[5] I. M. Lifshits, S. A. Gredeskul, L. A. Pastur (1988) Intro. to the Theory of Disordered Systems Wiley, New York.

[6] E. Orlandini, M. C. Tesi, and S. G. Whittington, J. Phys. A: Math. Gen. 33, 259 (2000).

[7] V. S. Pande, A. Yu. Grosberg, and T. Tanaka, Macromolecules 28, 2218 (1995).

[8] E. I. Shakhnovich and A. M. Gutin, Proc. Natl. Acad. 
Sci. USA 90, 7195 (1993).

[9] M. H. Hecht, J. S. Richardson, and D. C. Richardson, Science 249, 884 (1990).

[10] T. Enoki, et al. Physical Review Letters, 85, 5000 (2000).

[11] R. I. Dima, J. R. Banavar, M. Cieplak, and A. Maritan, Proc. Natl. Acad. Sci. USA 96, 4904 (1999).

[12] A. R. Davidson, K. J. Lumb, and R. T. Sauer, Nature Structural Biology 2, 856 (1995).

[13] U. Bastolla, P. Grassberger, cond-mat/0004169

[14] H. Li, R, Helling, C. Tang, and N. Wingreen Science 273, 666 (1996).

[15] R. Du, A. Yu. Grosberg, and T. Tanaka, Folding and Design 3, 203 (1998).

[16] M.Cieplak, N.Holter, A.Maritan, J.Banavar, J. Chem. Phys. 114, 1420, 2001.

[17] V. S. Pande, C. Joerg, A. Yu. Grosberg, and T. Tanaka, J. Phys. A 27, 6231 (1994). 